\title{
Considerations for Automating the Dissolution Test
}

\author{
G. Bryan Crist \\ Agilent Technologies, Cary, NC
}

e-mail:Bryan.crist@agilent.com

\begin{abstract}
A primary challenge for the pharmaceutical laboratory is to maximize throughput with optimum compliance. A technique-dependent test like dissolution is a primary target for implementing varying levels of automation, from semiautomated sampling equipment to fully robotic systems. Instrumentation flexibility will be required in the laboratory of the future to minimize the potential for error caused by manual intervention and to fully utilize the dissolution apparatus with automated sampling and on- and offline UV or HPLC analytical systems.

This article offers suggestions for determining the level of automation needed to obtain peak performance based on the product(s), laboratory requirements, and regulatory compliance associated with laboratory automation.
\end{abstract}

\section{THE VERSATILITY PARADOX}

Ever see an automated system sitting in the corner of the laboratory not being used or labeled "out of service?" One has to question why this happens.

The primary goal of automation is to have a system that will meet the needs of the laboratory. To accomplish this goal, we must evaluate laboratory workload and the type of dissolution samples to determine the most efficient configuration of automation enhancement. For instance, if the typical products tested are $80 \%$ immediate release, $75 \%$ are Apparatus 2 paddle methods, and $70 \%$ of the products require UV analysis, then we should focus automation on meeting these needs, possibly with an online UV dissolution system. If the laboratory also requires sample archiving for investigation purposes, an online autosampler should be added to the system.

Upon consideration of implementing automated dissolution equipment and methodology, one must review the product matrix for the particular analytical support required. When automated equipment is purchased without carefully targeting the specific product type or laboratory usage needs, the equipment may be insufficient or overly qualified for the task. The equipment acquired may be versatile well beyond normal testing requirements, may be more expensive, and may possess features that will never be used. Additional features can unnecessarily add to the burden of validation and qualification, and in some cases can be such an obstacle that the automated system implementation is delayed or never reaches the intended level of utilization. This results in the versatility paradox of understanding that what we "want" in a system may not be what we actually "need." The ability to have a system that completely automates sampling and the analytical finish is alluring, yet can unnecessarily increase complexity, cost, and in the end, efficiency.

\section{THE CONCEPT OF DISSOLUTION AUTOMATION}

Dissolution automation begins with an evaluation of typical dissolution test requirements that involve human manipulation, processing, and decision. If a series of similar steps are routinely performed, and if the system will conduct the same type of analysis, automated methods of analysis may be more desirable than manual methods for greater efficiency and precision. The dissolution test is simply broken down to sample preparation using the apparatus followed by UV or HPLC analysis. These two events are separated by filtration, which stops the dissolution process and clarifies the sample for analytical measurement.

Dissolution consists of a series of unit operations that are quite technique-dependant and may cause inconsistencies in test results when performed manually. While the dissolution test may be subdivided into many individual steps, five major components will be discussed regarding automation: setup, dissolution, sampling, analysis and data reduction, and finally, cleanup and changeover. Since the entire dissolution test is a series of these five components, any one may be performed manually or automatically. If only one of the components is automated, that step is considered semiautomated. If all five of the components are automated sequentially, this approaches total automation. Before evaluating advantages and limitations of automating the dissolution test, we must first evaluate the five components of the test.

\section{Setup}

In dissolution testing, there will always be some human intervention in terms of making media and setting up the apparatus. Media preparation is usually accomplished by manual preparation, degassing, measurement, and documentation. 
Media dispensing may be performed gravimetrically or volumetrically, though automated systems usually rely on gravimetric means to deliver media. Automated dispensing systems will generally preheat and degas media but must be capable of cleaning, purging, and calibrating to ensure accuracy within $\pm 1 \%$ as required in the USP. Additionally, transfer lines must be checked to ensure that they are not crimped and that the connections are tightly sealed.

The essential setup task of dosage handling to protect the sample from humidity and temperature before the start of the run must also be performed. Automated dosage delivery systems must also be capable of handling capsules with sinker devices attached.

During the setup phase, automated systems should be evaluated that components are clean will be set up with the proper method and filters.

\section{The Dissolution Apparatus}

The dissolution apparatus is the sample preparation device that determines the performance of the drug release under very controlled conditions of time, temperature, agitation, and volume control. Sample integrity must be maintained when samples are removed from the vessel and filtered. After filtration, the analytical phase of testing occurs where the amount of active pharmaceutical ingredient (API) in solution at a specific time is determined quantitatively.

One of the important requirements of the dissolution test is maintenance of the apparatus as described in the USP harmonized dissolution chapter. Whenever a dissolution method or procedure states that USP Apparatus 1 basket method is used, the detailed configuration of the apparatus must be followed. We may use apparatus modifications only if they have been properly validated for use. For example, the use of an o-ring attachment and modified basket in an automated method must be validated to show that it is equivalent to the official clip design. Sampling probes left in the vessel during a run must be verified to document that the modified apparatus will produce results equivalent to apparatus as described in USP without the probes present.

Validation is a process that verifies the equivalence of one procedure to another. If there is a difference of greater than 2-3\% between the apparatus described in USP $<711>$ and the modified apparatus, then the change is unacceptable. Likewise, temperature must be monitored to assure proper conditions throughout the period of testing.

Dropping the dosage form into non-rotating media, as required in the USP, may also be challenging for some systems. Automated systems may have multiple ports for tablet introduction, sample withdrawal, and addition and removal of media. The evaporation covers for automated systems should prevent the possibility of evaporative loss during the test.

\section{Dissolution Sampling}

Most of the "hands-on" time that it takes to perform a dissolution test is spent preparing for and cleaning up after the dissolution test. However, the most common type of semiautomation that is applied to the dissolution test is the addition of an automatic sampling system.

Automatic sampling may provide gains in precision over manual sampling techniques since the location, timing, and filtration aspects are controlled and executed without variance from analysts, vessels, or both. While time saving is vitally important, the focus on dissolution apparatus with automated sampling indicates that one of the primary goals for automation is reduction of variability in addition to time. Automated systems must be validated to ensure that the proper sampling method is reproduced by an automated system.

Other requirements include the need to rinse and purge sample lines, load and condition filters with the proper amount of media, lower the sampling cannula to the proper position, and validate that hydrodynamic factors are negligible if cannulas remain in the vessel throughout the test. Sampling lines must be routinely checked to ensure they are not damaged or crimped and are properly rinsed. Transfer tubing should not adsorb active drug product, and cleaning methods must be appropriately validated to ensure sample integrity.

Automated sampling systems must be able to pull samples precisely at the correct time interval with accurate volume, and systems that replace media must have correspondingly accurate and preheated replacement volumes. Extended-release samples or those that will not be immediately analyzed must be maintained in sealed test tubes or HPLC vials to negate concentration issues due to evaporation.

\section{Analysis and Data Reduction}

Once filtered samples are obtained, the dissolution process ends, and the samples are then evaluated typically by UV-vis spectrophotometry or HPLC. Depending on the level of automation, the analytical finish may be on- or offline. Justification of automated equipment should be based on obtaining the maximum utilization of the dissolution apparatus, sampling equipment, and the UV or HPLC system.

Automated ultraviolet, or UV-vis, systems provide an economical and faster alternative to chromatographic methods, allowing an increased number of timepoints due to the short analysis time. Advanced multicomponent UV-vis spectroscopic systems have the potential for being less costly than HPLC automated systems due to their simplicity. These systems are ideally suited for fiber-optic analysis, but often have not gained acceptance since true separation of components does not occur as with HPLC.

Fiber-optic UV-vis spectroscopic systems employ several advantages over traditional analysis. The capa- 
bility of bringing light to the vessel rather than bringing the sample to the spectrophotometer eliminates cross-contamination and fluidics problems involved in the sample transmission process. These systems, when incorporated with adequate software, provide a tremendous number of data points for detailed dissolution profiles. While the future is very promising for fiber-optic systems, the integrity of the dissolution hydrodynamics must be preserved because of the size of the fiber-optic probes. Increased technology in microprobes or suspending the probes just under the surface between time points have greatly reduced problems associated with hydrodynamics.

High performance liquid chromatography (HPLC) has traditionally been implemented to provide an analytical finish for dissolution samples with multiple components or excipients that interfere with measurement.

Many HPLC systems are considered "semiautomated" since the methods are loaded along with a sample sequence that corresponds to standard and sample vial locations. After system suitability conditions are met, samples are automatically injected, integrated, and batchprocessed to provide the analytical concentration of the dissolution samples. Some systems may reconstruct the sample concentrations to provide a full dissolution report complete with a profile of the dissolution run.

Through the transfer of the sample to the analytical instrument that determines the concentration, the sample must not be compromised or altered to provide an inaccurate measurement. Automated analytical finishes will determine system suitability, measure and calculate the amount of active drug substance dissolved, perform statistical manipulations for comparing data, graphically display rates of release in a dissolution profile, and eventually store and archive the data after a printed report is obtained.

Although online HPLC systems exist, instrument utilization must be considered. Will the HPLC be relatively idle while a dissolution run is in progress? After all the samples are processed on the HPLC, will the dissolution apparatus be idle? Offline dissolution and HPLC testing is likely the most convenient way to accumulate samples from several dissolution apparatus and combine them into a single long run of HPLC sample sets. In this manner, the HPLC is fed by several dissolution apparatus to approach full utilization of the laboratory equipment. Offline HPLC software is also available to process the samples into a full dissolution report with profile.

\section{Cleanup and Changeover}

Cleanup and changeover may begin before samples are measured, but the samples should not be discarded in the event that out of specification (OOS) or suspect data is obtained. Samples should also be read and results calculated while the samples are still within their period of analytical stability in the event they must be reread due to a suspect result. Once the dissolution apparatus has finished its run, the test is terminated. After the test, media must be removed from the dissolution vessels. Dissolution vessels must be thoroughly cleaned as validated through manual or automatic clean-in-place (CIP) methods. In preparation for testing the next samples, all product-contact equipment, including evaporation covers, cannula transfer lines, pump tubes, flow cells, valves, and sample injectors must be properly cleaned to eliminate the risk of contaminating the next product to be analyzed.

\section{UNDERSTANDING YOUR PRODUCT}

Review of the product matrix may reveal, for instance, that seventy percent of the products in a particular laboratory are tablets run with the paddle method and analyzed using a UV-vis spectrophotometer. This simple, yet criti$\mathrm{cal}$, information alone determines that the system requirements do not need to be configured for basket methods or an HPLC analytical finish. Additional information on media constraints, filtering needs, and handling dilution offers further insight into product testing requirements.

\section{UNDERSTANDING YOUR LABORATORY AND RESOURCES}

Insufficient due diligence for determining the best system for a laboratory is often the reason that automated equipment is cast aside or sits underutilized rather than meeting its full potential. For example, highly automated online robotic systems work very well in supporting a high throughput laboratory testing numerous batches of similar products. However, a laboratory testing a large number of different products everyday may be better off with a semiautomated approach to achieve efficiency.

Just as understanding laboratory demands influences automated equipment requirements, so does the input of operations supporting the laboratory in terms of preventative maintenance, qualification, calibration, validation, laboratory information management, and QA. If a laboratory purchases automated equipment without consulting other departments that will provide additional internal support, delays implementing or sustaining the use of automated equipment can result.

\section{DISSOLUTION TEST AUTOMATION SUMMARY}

When we look at the operational capabilities to determine which take the most hands-on time, the setup and cleanup may reflect the greatest time requirement. It is quite interesting, however, that sample collection with an autosampler is the main area in which semiautomation is applied to dissolution testing.

While we often look at automation as buying time to perform other analytical tasks, it cannot be denied that our goal in the cGMP laboratory is to provide dependable, accurate, and precise testing on equipment that meets ICH harmonized pharmacopeial and regulatory requirements. When properly employed, upfront investments 
in automated dissolution equipment and the validation of automated methods are eventually realized not only in benefits and cost savings, but in reliable testing that is reproducible among analysts and across laboratories. The key to full utilization and a successful investment is, of course, a result of proper evaluation of product and laboratory requirements from the start.

\section{BIBLIOGRAPHY}

1. $<711>$ Dissolution. In The United States Pharmacopeia and National Formulary USP 36-NF 31; The United States Pharmacopeial Convention, Inc.: Rockville, MD, 2013.

2. <1225> Validation of Compendial Methods. In The United States Pharmacopeia and National Formulary USP 36-NF 31; The United States Pharmacopeial Convention, Inc.: Rockville, MD, 2013.
3. Dissolution Testing of Immediate Release Solid Oral Dosage Forms; Guidance for Industry; U.S. Department of Health and Human Services, Food and Drug Administration, Center for Drug Evaluation and Research (CDER), U.S. Government Printing Office: Washington, DC, 1997.

4. Validation of Chromatographic Methods; Reviewer Guidance; U.S. Department of Health and Human Services, Food and Drug Administration, Center for Drug Evaluation and Research (CDER), U.S. Government Printing Office: Washington, DC, 1994.

5. Palmieri, A. Dissolution Theory, Methodology, and Testing; Dissolution Technologies, Inc.: Hockessin, DE, 2007.

6. Dissolution Discussion Group Home Page. http://www. dissolution.com (accessed April 11, 2013). 